would need to be analyzed by TP53 IHC, only $46(7.7 \%)$ by MMR IHC and 286 (48.1\%) POLE sequencing reactions.

Conclusion* Application of the 2021 molecular risk groups is feasible and shows significant differences in survival. IHC for TP53 and MMR and applying POLE sequencing is only needed in selected cases and leads to shifting risk groups both upward and downward for a sizeable number of patients. It is possible to significantly reduce the number of analyses required to implement the classification if resources are limited.

\section{COMBINED USE OF ICG AND TECHNETIUM DOES NOT IMPROVE SENTINEL LYMPH NODE DETECTION IN ENDOMETRIAL CANCER}

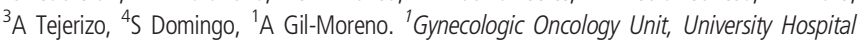
Vall d'Hebron, Vall d'Hebron Barcelona Hospital Campus and Autonomous University of Barcelona (UAB); ${ }^{2}$ Gynecologic Oncology Unit, University Hospital of Bellvitge (IDIBELL), Barcelona; ${ }^{3}$ Gynecologic Oncology Unit, University Hospital 12 de Octubre, Madrid; ${ }^{4}$ Gynecologic Oncology Unit, University Hospital La Fe, Valencia

\subsection{6/ijgc-2021-ESG0.203}

Introduction/Background* There is scarce evidence available about the benefit of combining technetium $(99 \mathrm{mTc})$ and indocyanine green (ICG) for sentinel lymph node (SLN) biopsy in endometrial cancer. The aim of this study was to compare the overall and bilateral pelvic detection rates of sentinel lymph nodes in two retrospective cohorts: ICG exclusive vs. combined ICG $+99 \mathrm{mTc}$.

Methodology Multicentre retrospective study (November 2015June 2020) including patients diagnosed with endometrial atypical hyperplasia or early-stage endometrial carcinoma who underwent sentinel lymph node biopsy by cervical injection of ICG with or without $99 \mathrm{mTc}$ in four different referral centers in Spain.

Result(s)* A total of 180 patients were included, 51\% (n=92) in ICG group and $49 \%(n=88)$ in ICG $+99 \mathrm{mTc}$ group. Eighty-six percent of the patients presented endometrioid histology, and over ninety-nine percent of the procedures were performed by a minimally invasive approach. Both groups were comparable regarding their basal characteristics, except for a higher body mass index in ICG $+99 \mathrm{mTc}$ group and a bigger proportion of robotic-assisted procedures in ICG group.

Overall detection rate was $92.8 \%$ and similar between groups (ICG: $94.6 \%$ vs ICG+99mTc: 90.9\%, p=.34). No significant differences were observed neither in bilateral pelvic nor aortic mapping rate. When $99 \mathrm{mTc}$ was used, surgical procedures were significantly longer. In $6.7 \%$ of patients, at least one positive SLN was found (ICG:9.8\% vs ICG+99mTc:3.4\%, $\mathrm{p}=.164)$. Empty node packet rates and number of SLNs retrieved per patient were also similar between cohorts.

Conclusion* Combining preoperative $99 \mathrm{mTc}$ to intraoperative ICG did not improve SLN detection in endometrial cancer, but resulted in longer procedures.

\section{SENTINEL LYMPH NODE BIOPSY IN OPEN SURGERY FOR GYNAECOLOGICAL MALIGNANCIES}

L Bocciolone, A Bergamini, R Cioffi, F Cantatore, E Rabaiotti, F Sgalambro, G Sabetta, G Candotti*. San Raffaele Hospital, Obstetrics and Gynecology, Milano, Italy

10.1136/ijgc-2021-ESG0.204
Introduction/Background* Sentinel node biopsy has been introduced as alternative to lymph node dissection for lymph node staging for endometrial cancer patients. This surgical video has the objective to show the feasibility of Indocyanine Green (ICG) sentinel node sampling using SPY Portable Handheld Imaging System during open endometrial cancer surgery.

Methodology 72 years old patient with diagnosis of endometrioid G2 endometrial cancer underwent open surgery due to anesthesiological and surgical contraindications.

Result(s)* Laparotomy with total extrafascial hysterectomy, bilateral salpingoophorectomy and bilateral lymph node biopsy was performed. Operative time was 110 minutes and blood loss was 200cc. Patient was discharged after 4 days without any complication.

Conclusion* The use of SPY Portable Handheld Imaging System in open surgery seems to be a feasible and useful tool for the detection of SLN in endometrial cancer patients underwent open surgery.

\section{METASTATIC ENDOMETRIAL CARCINOMA ARISING FROM ADENOMYOSIS, PRESENTING WITH DEEP VEIN THROMBOSIS AND VISUAL IMPAIRMENT; CASE REPORT}

${ }^{1} \mathrm{~L}$ Kovač*, ${ }^{2} \mathrm{M}$ Pavlova Bojadžiski, 2;3E Skof, ${ }^{3 ; 4} \mathrm{~L}$ Meglič. ${ }^{1}$ University Medical Centre Ljubljana, Department of gynaecology , Ljubljana, Slovenia; ${ }^{2}$ Institute of Oncology Ljubljana, Department of Medical Oncology, Ljubljana, Slovenia; ${ }^{3}$ Faculty of Medicine, Slovenia; ${ }^{4}$ University Medical Centre Ljubljana , Department of Gynaecology , Ljubljana, Slovenia

\subsection{6/ijgc-2021-ESGO.205}

Introduction/Background* Endometrioid carcinoma arising in adenomyosis (EC-AIA) is a rare entity. We present a case of an advanced stage presenting with deep vein thrombosis (DVT) and visual impairment.

Methodology 42-year old patient presented to our institution, due to DVT of left femoral vein. During hospitalisation, an abdominal ultrasound showed a uterine tumour, resembling a myoma. Increased $\mathrm{Ca} 125$ and $\mathrm{Ca}$ 15-3 were observed. No subsequent diagnostic procedures were performed, a follow up visit with personal gynaecologist was recommended. In the following weeks, she noticed visual impairment and trouble with understanding. Cranial MRI showed metastatic lesions and possible meningeal carcinosis. Patient was readmitted. Abdominal computer tomography (CT) showed a pelvic necrotic formation between right ovary and uterus, with pathologic iliac and paraaortic lymph nodes. Multidisciplinary team indicated primary debulking surgery. Before the procedure, she underwent coloscopy and gastroscopy without pathologic findings.

Median laparotomy with hysterectomy, omentectomy, appendectomy and debulking of pelvic and paraaortic lymph nodes were performed. Pathohistological report showed a necrotic tumour, originating in the myometrium on the right lateral side of the uterus and infiltrating the right salpinx and ovary. Microscopic evaluation revealed endometrioid adenocarcinoma, grade 1, originating in myometrium, without involvement of endometrium. Positive right obturator and paraaortic lymph nodes did not involve the capsule. Immunohistochemistry showed positive oestrogen and progesterone receptors and p53-wt expression. Additional genetic analysis was done, due to loss of MSH6 protein. Due to probable cranial involvement, stage was set as FIGO IVB. Subsequent treatment consisted of palliative radiotherapy of the cranium, followed by 
introduction of letrozole as maintenance therapy. Follow up imaging studies showed no signs of disease progression. At the last check up, the patient was in good condition, without specific complaints.

Result(s)*

Conclusion* Mechanism of EC-AIA is not well understood. As in our case, the tumour can mimic benign uterine lesions and postpone a proper diagnosis. This can lead to advanced stage disease with uncommon clinical presentation. Few studies have described the molecular mechanism of adenomyosis formation. It has been suggested that loss of heterozygosity in the DNA mismatch repair family is associated with adenomyosis and its pathogenesis. Better understanding of the molecular and immunologic drivers of response and resistance will be critical in management of EC-AIA.

\section{MINIMALLY INVASIVE VERSUS OPEN HYSTERECTOMY IN HIGH-RISK ENDOMETRIAL CANCER: A PROPENSITY SCORE MATCHING ANALYSIS}

${ }^{1} \mathrm{~B}$ Segarra Vidal ${ }^{*},{ }^{2} \mathrm{~A}$ Zorrills-Vaca, ${ }^{3} \mathrm{G}$ Dinoi, ${ }^{4} \mathrm{~V}$ Študent, ${ }^{5} \mathrm{~N}$ Agustí, ${ }^{6 ; 7} \mathrm{~A}$ Llueca, ${ }^{8} \mathrm{~A}$ Mariani, ${ }^{9} \mathrm{P}$ Ramirez. ${ }^{1} \mathrm{Hospital}$ Universitario y Politécnico de La Fe, Gynecologic Oncology, València, Spain; ${ }^{2}$ MD Anderson Main Building, Houston, USA; ${ }^{3}$ Gemelli, Roma, Italy; ${ }^{4}$ Mayo Clinic, Rochester, USA; ${ }^{5}$ Hospital Clínic de Barcelona, Barcelona, Spain; ${ }^{6}$ Hospital General Universitari de Castelló, Castelló de la Plana, Spain; ${ }^{7}$ Gynecology Oncology Unit Hospital General Universitario de Castellon. Departamento de Medicina. Universitat Jaume I (UII). , Spain; ${ }^{8}$ Mayo Clinic Rochester MN, Rochester, USA; ${ }^{9}$ The University of Texas MD Anderson Cancer Center, Houston, USA

\subsection{6/ijgc-2021-ESG0.206}

Introduction/Background* Randomized trials have shown comparable oncologic outcomes between open versus minimally invasive surgery for endometrial cancer. Limited data is available specifically in patients with high-risk disease. The aim of this study was to compare disease-free survival, overall survival, and recurrence rates between minimally invasive surgery versus open surgery in patients with high-risk endometrial cancer.

Methodology This was a multicentric, propensity score matched study of patients with high-risk endometrial cancer who underwent total abdominal hysterectomy, bilateral salpingo-oophorectomy and staging between January 1999 and June 2016 at two referral cancer centers. High-risk endometrial cancer included uterine grade 3 endometrioid, serous carcinoma, clear cell carcinoma, and undifferentiated carcinoma or carcinosarcoma with any degree of myometrial invasion. Patients were categorized a priori into two groups based on the surgical approach, propensity scores were calculated based on potential confounders and then both groups were matched in a 1:1 fashion using the nearest neighbor technique. Cox hazard regression analysis was used to evaluate effect of surgical technique on survival.

Result(s)* A total of 626 patients were eligible, of which 263 patients underwent minimally invasive surgery and 363 open surgery. The median age was 67 years (IQR 60-74), and the median body mass index was $30.5 \mathrm{~kg} / \mathrm{m}^{2}$ (IQR 25.5-35.8). After matching, both groups had 185 matched pairs with comparable demographics and clinical characteristics. In the matched cohort, there were no differences in disease-free survival rates at 5 -years between open surgery $(53.4 \%$ [95\% CI 45.6-60.5\%]) and minimally invasive surgery $(54.6 \%$ [95\%CI 95\% 46.6-61.8]; $\mathrm{P}=0.82$ ). Minimally invasive surgery was not associated with worse disease-free survival (HR 0.85 , 95\% CI 0.63 to $1.16 ; \mathrm{P}=0.30$ ), overall survival (HR 1.04, $95 \%$ CI 0.73 to $1.48, \mathrm{P}=0.81)$, or recurrence rate (HR 0.99 ; CI 95\% 0.69-1.44; $\mathrm{P}=0.99$ ) compared to open surgery. Use of uterine manipulator was not associated with worse disease-free survival (HR 1.01, 95\% CI 0.65 to 1.58 , $\mathrm{P}=0.96$ ), overall survival (HR 1.18 , 95\% CI 0.71 to 1.96 , $\mathrm{P}=0.53)$, or recurrence rate $(\mathrm{HR}, 1.12$; CI $95 \% 0.67$ to 1.87 ; $\mathrm{P}=0.66$ )
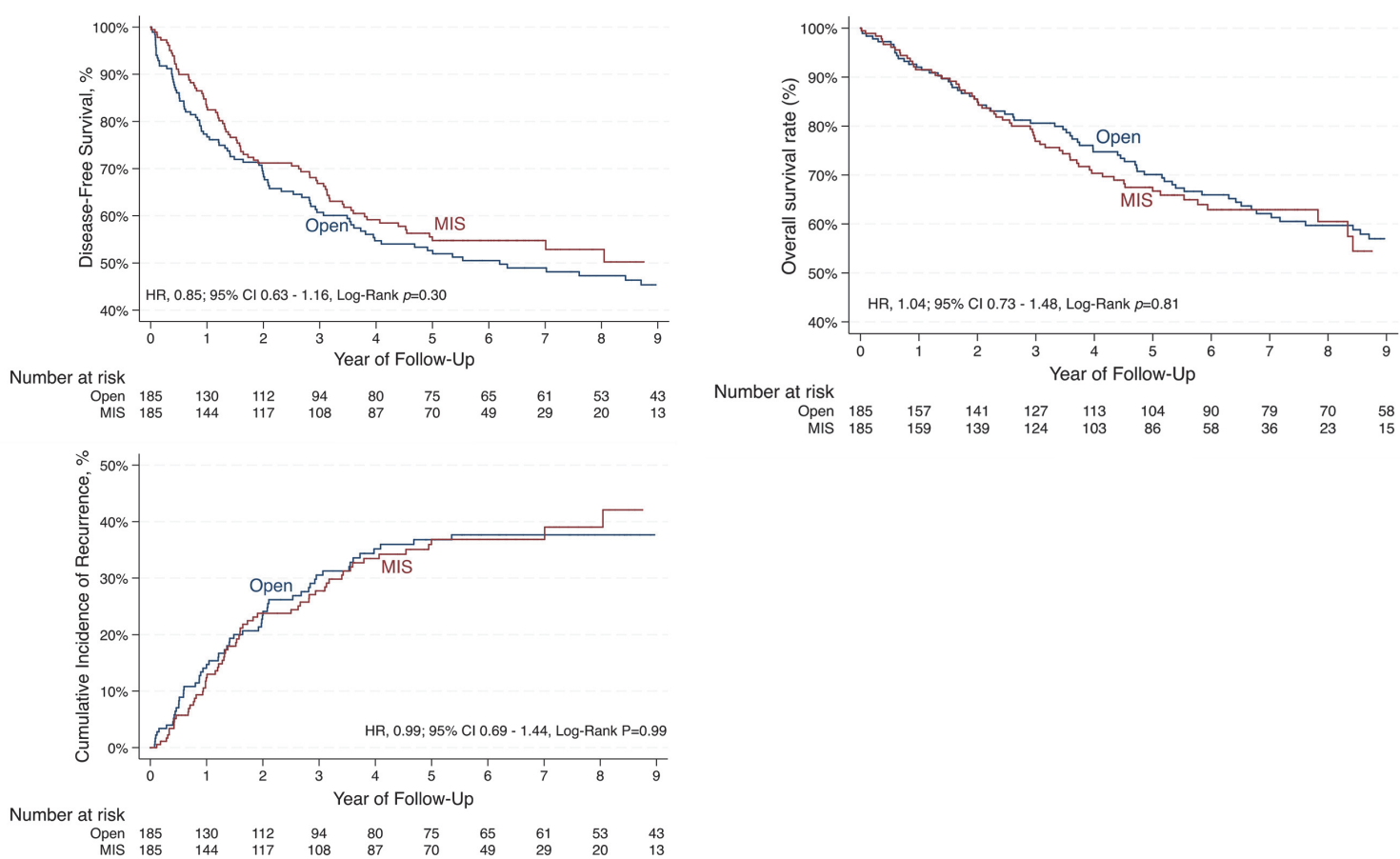

Abstract 864 Figure 1 